


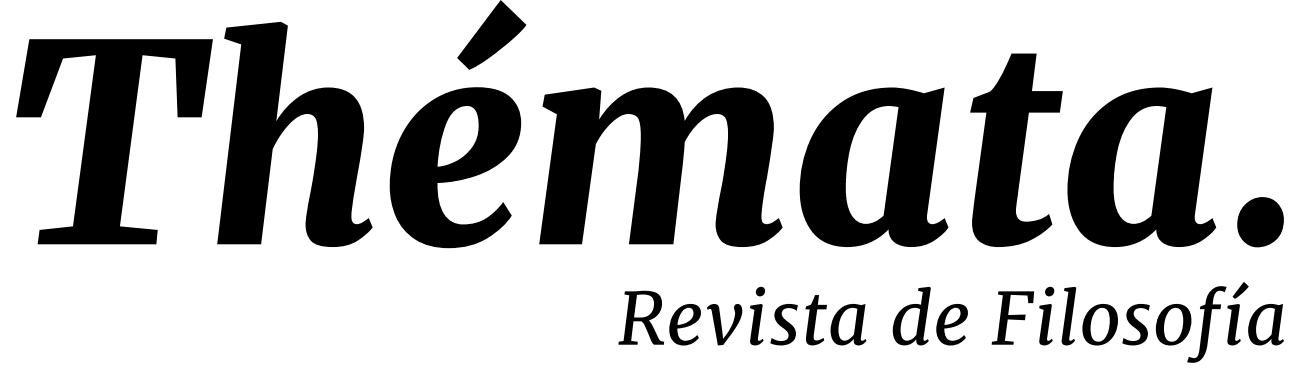

\section{La transmisión filosófica. Pensamiento de Javier Hernández-Pacheco Sanz}

Alejandro Martín Navarro (Coord.)

ESPECIAL THÉMATA

2022

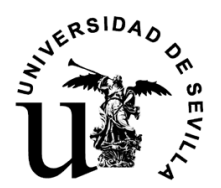

ISSN 0212-8365

e-ISSN 2253-900X

DOI: $10.12795 /$ themata

revistascientificas.us.es/index.php/themata

https://editorial.us.es/es/revistas/themata-revista-de-filosofia 
Thémata. Revista de Filosofía.

Número Extra

Thémata. Revista de Filosofía nace en el año 1983 con la intención de proporcionar a quienes investigan y producen en filosofía un cauce para publicar sus trabajos y fomentar un diálogo abierto sin condicionamientos ideológicos. En sus inicios participaron en el proyecto las Universidades de Murcia, Málaga y Sevilla, pero pronto quedaron como gestores de la revista un grupo de docentes de la Facultad de Filosofía de la Universidad de Sevilla.

Una preocupación constante de sus realizadores ha sido fomentar los planteamientos interdisciplinares. La revista ha estado abierta siempre a colaboradores de todas las latitudes y ha cubierto toda la gama del espectro filosófico, de lo que constituye una buena prueba la extensa nómina de autores que han publicado en sus páginas. En sus páginas pueden encontrarse trabajos de todas las disciplinas filosóficas: Historia de la Filosofía, Metafísica, Gnoseología, Epistemología, Lógica, Ética, Estética, Filosofía Política, Filosofía del Lenguaje, Filosofía de la Mente, Filosofía de la Ciencia, Filosofía de la Historia, Filosofía de la Cultura, etc. También ha querido ser muy flexible a la hora de acoger nuevos proyectos, fomentar discusiones sobre temas controvertidos y abrirse a nuevos valores filosóficos. Por esta razón, los investigadores jóvenes siempre han encontrado bien abiertas las puertas de la revista.

\section{Equipo editorial / Editorial Team Bases de Datos y Repertorios}

Director honorario

Jacinto Choza Armenta

jchoza@us.es

Director

Fernando Infante del Rosal

finfante@us.es

Director Adjunto

José Manuel Sánchez López

themata@us.es

Subdirectores

Jesús Navarro Reyes

jnr@us.es

Inmaculada Murcia Serrano

imurcia@us.es

Jesús de Garay

jgaray@us.es

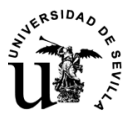

Facultad de Filosofía. Universidad de Sevilla

Departamento de Estética e Historia de la

Filosofía

Departamento de Filosofía y Lógica y Filosofía

de la Ciencia

Departamento de Metafísica y Corrientes

Actuales de la Filosofía, Ética y Filosofía Política

Camilo José Cela s/n, 41018 Sevilla (España)

e-mail: themata@us.es
Bibliográficas internacionales

Emerging Sources Citation Index (Web of

Science Group-Clarivate Analytics)

Dialnet (España)

Francis, Philosophie. INIST-CNRS (France)

Philosopher's Index (Bowling Green, OH, USA)

Directory of Open Access Journals (DOAJ)

Repertoire Bibliographique de Philosophie

(Louvain, Belgique)

Ulrich's Internacional Periodicals Directory

(New York, USA)

DialogJournalNameFinder (Palo Alto, CA, USA)

Periodicals Index Online (Michigan, USA)

Index Copernicus World of Journals

Gale-Cengage Learning-Informe Académico

Academic Journal Database

DULCINEA

Google Scholar

Electra

Bulletin signaletique. Philosophie, CNRS

(France)

Bibliográficas nacionales

ISOC - Filosofía. CINDOC (España)

De evaluación de la calidad de revistas

CARHUS Plus

ERIH PLUS Philosophy (2016)

REDIB

Latindex 2.0

MIAR

CIRC

DICE

Política editorial y directrices para autores/as, al final de la revista.

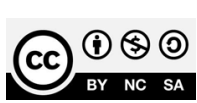


Thémata. Revista de Filosofía.

\section{Consejo Editor / Editorial Board}

\section{ARGENTINA}

Flavia Dezzuto, Universidad Nacional de Córdoba

ALEMANIA

Alberto Ciria, Munich

\section{CANADÁ}

Óscar Moro, University of New Found Land

CHILE

Mariano De la Maza, Universidad Católica de Chile

José Santos Herceg, Universidad de Santiago de Chile

\section{COLOMBIA}

Martha Cecilia Betancur García, Universidad de Caldas

Víctor Hugo Gómez Yepes, Universidad Pontificia Bolivariana

Gustavo Adolfo Muñoz Marín, Universidad Pontificia Bolivariana

\section{ESPAÑA}

Alfonso García Marqués, Universidad de Murcia

Antonio De Diego González, Universidad de Sevilla

Avelina Cecilia Lafuente, Universidad de Sevilla

Carlos Ortiz Landázuri, Universidad de Navarra

Celso Sánchez Capdequí, Universidad Pública de Navarra

Elena Ronzón Fernández, Universidad de Oviedo

Enrique Anrubia, Universidad CEU Cardenal Herrera

Federico Basáñez, Universidad de Sevilla

Fernando Wulff, Universidad de Málaga

Fernando M. Pérez Herranz, Universidad de Alicante

Fernando Pérez-Borbujo, Universitat Pompeu Fabra

Francisco Rodríguez Valls, Universidad de Sevilla

Ildefonso Murillo, Universidad Pontificia de Salamanca

Irene Comins Mingol, Universitat Jaume I Jacinto Rivera de Rosales Chacón, † UNED Joan B. Llinares, Universitat de València Jorge Ayala, Universidad de Zaragoza José Manuel Chillón Lorenzo, Universidad de Valladolid
Juan García González, Universidad de Málaga

Juan José Padial Benticuaga, Universidad de Málaga

Luis Miguel Arroyo Arrayás, Universidad de Huelva

Ma Luz Pintos Peñaranda, Universidad de Santiago de Compostela

Marcelo López Cambronero, Instituto de Filosofía Edith Stein

María del Carmen Paredes, Universidad de Salamanca

Octavi Piulats Riu, Universitat de Barcelona

Óscar Barroso Fernández, Universidad de Granada

Pedro Jesús Teruel, Universitat de València

Ramón Román Alcalá, Universidad de Córdoba

Ricardo Parellada, Universidad Complutense de Madrid

Sonia París Albert, Universitat Jaume I

Tomás Domingo Moratalla, UNED

\section{ESTADOS UNIDOS}

Witold Wolny, University of Virginia)

Thao Theresa Phuong Phan, University of Maryland

\section{REINO UNIDO}

Beatriz Caballero Rodríguez, University of Strathclyde

ITALIA

Luigi Bonanate, Università di Torino

\section{MÉXICO}

Rafael De Gasperín, Instituto Tecnológico de Monterrey

Julio Quesada, Universidad Veracruzana

Adriana Rodríguez Barraza, Universidad Veracruzana

PERÚ

Ananí Gutiérrez Aguilar, Universidad Nacional de San Agustín de Arequipa y Universidad Católica de Santa María

Nicanor Wong Ortiz, Universidad San Ignacio de Loyola

\section{PORTUGAL}

Yolanda Espiña, Universidade Católica Portuguesa

\section{TURQUÍA}

Mehmet Özkan, SETA Foundation for Political, Economic and Social Research 
Thémata. Revista de Filosofía.

\section{Comité Científico Asesor / Advisory Board}

ARGENTINA

Graciela Maturo, Universidad de Buenos Aires - CONICET

Jaime Peire, Universidad Nacional de Tres de Febrero- CONICET

\section{ALEMANIA}

Tomás Gil, Freie Universität Berlin

Fernando Inciarte, $\dagger$ Westfälische WilhelmsUniversität

Otto Saame, † Universität Mainz

\section{BULGARIA}

Lazar Koprinarov, South-West University 'Neofit Rilski'

CHILE

Carla Corduá, Universidad de Chile

Roberto Torreti, Universidad de Chile

COLOMBIA

Carlos Másmela, Universidad de Antioquía Fernando Zalamea, Universidad Nacional de Colombia

\section{ESPAÑA}

Agustín González Gallego, Universitat de Barcelona

Alejandro Llano, Universidad de Navarra Andrés Ortiz-Osés, Universidad de Deusto Ángel D'ors, † Universidad Complutense de Madrid

Antonio Hermosa Andújar, Universidad de Sevilla

Carlos Beorlegui Rodríguez, Universidad de Deusto

Concha Roldán Panadero, Instituto de Filosofía, CCHS-CSIC

Daniel Innerarity Grau, Ikerbasque, Basque Foundation for Science

Francisco Soler, Universidad de Sevilla Ignacio Falgueras, Universidad de Málaga Javier San Martín, UNED

Jesús Arellano Catalán, † Universidad de Sevilla

Joaquín Lomba Fuentes, Universidad de Zaragoza

Jorge Vicente Arregui, † Universidad de Málaga José María Prieto Soler, † Universidad de Sevilla José Rubio, Universidad de Málaga Juan Antonio Estrada Díaz, Universidad de Granada

Juan Arana Cañedo-Argüelles, Universidad de Sevilla
Luis Girón, Universidad Complutense de Madrid

Manuel Fontán Del Junco, Fundación March

Manuel Jiménez Redondo, Universitat de València

Marcelino Rodríguez Donís, Universidad de Sevilla

Miguel García-Baró López, Universidad Pontificia Comillas

Modesto Berciano, Universidad de Oviedo

Pascual Martínez-Freire, Universidad de Málaga

Rafael Alvira, Universidad de Navarra

Teresa Bejarano Fernández, Universidad de Sevilla

Vicente San Félix Vidarte, Universitat de València

\section{ESTADOS UNIDOS}

Lawrence Cahoone, University of Boston

FRANCIA

Nicolás Grimaldi, Université Paris IV-Sorbonne

\section{PARAGUAY}

Mario Ramos Reyes, Universidad Católica de Asunción

\section{REINO UNIDO}

Alexander Broadie, University of Glasgow

ISRAEL

Marcelo Dascal, † Tel Aviv University

ITALIA

Massimo Campanini, Università di Napoli l'Orientale

Maurizio Pagano, Università degli Studi del Piamonte Orientale. Amedeo Avogadro

JAPóN

Juan Masiá, Sophia University, Tokio

\section{MÉXICO}

Jaime Méndez Jiménez, Universidad Veracruzana

Ana Laura Santamaría, Instituto Tecnológico de Monterrey

Héctor Zagal, Universidad Panamericana

VENEZUELA

Seny Hernández Ledezma, Universidad Central de Venezuela

Thémata. Revista de Filosofía agradece la labor realizada desde su inicio por sus anteriores directores: Jacinto Choza, Juan Arana y Jesús de Garay. 


\section{Índice.}

\section{LA TRANSMISIÓN FILOSÓFICA. PENSAMIENTO DE JAVIER HERNÁNDEZ-}

PACHECO SANZ_ESPECIAL THÉMATA

9 El acto filosófico. En recuerdo de Javier Hernández-Pacheco Sanz Fernando Infante del Rosal

14 Presentación

Alejandro Martín Navarro (Coord.)

SEMBLANZAS Y RECUERDOS_JAVIER HERNÁNDEZ-PACHECO SANZ,

TRANSMISIÓN Y ENSEÑANZA DE LA FILOSOFÍA

19 Treinta y siete años con Pacheco. Variaciones sobre el cristianismo Jacinto Choza Armenta

$51 \quad$ Historia de un hombre sabio y bueno Juan Arana Cañedo-Argüelles

$54 \quad$ Una fuerza de la naturaleza José Manuel Sánchez López

58 In Memoriam a Javier Hernández-Pacheco Urbano Ferrer Santos

65 Recordando al profesor Javier Hernández-Pacheco Esperanza María Domínguez Sabido

OBRA Y PENSAMIENTO DE JAVIER HERNÁNDEZ-PACHECO SANZ_ARTÍCULOS

$71 \quad$ Una conversación pendiente Jesús de Garay Suárez-Llanos

87 Un viaje de ida y vuelta: de cómo el refugio se torna apertura. El proyectar(se) como apertura a lo Otro Elisa Fernández Bascones

107 Max Horkheimer y el anhelo de lo completamente Otro. (A propósito de la interpretación de la filosofía de Max Horkheimer de Javier Hernández-Pacheco) José Manuel Panea Márquez 
Thémata. Revista de Filosofía.

124 Javier Hernández-Pacheco: fe y filosofía

Lidia Romero Sánchez

$141 \quad$ La plenitud hacia la que tendemos: historia de una discusión truncada.

Francisco José Soler Gil

161 Tábula gratulatoria.

163 Política editorial.

166 Directrices para autores/as.

ILUSTRACIÓN DE CUBIERTA: Javier Infante 
Thémata. Revista de Filosofía.
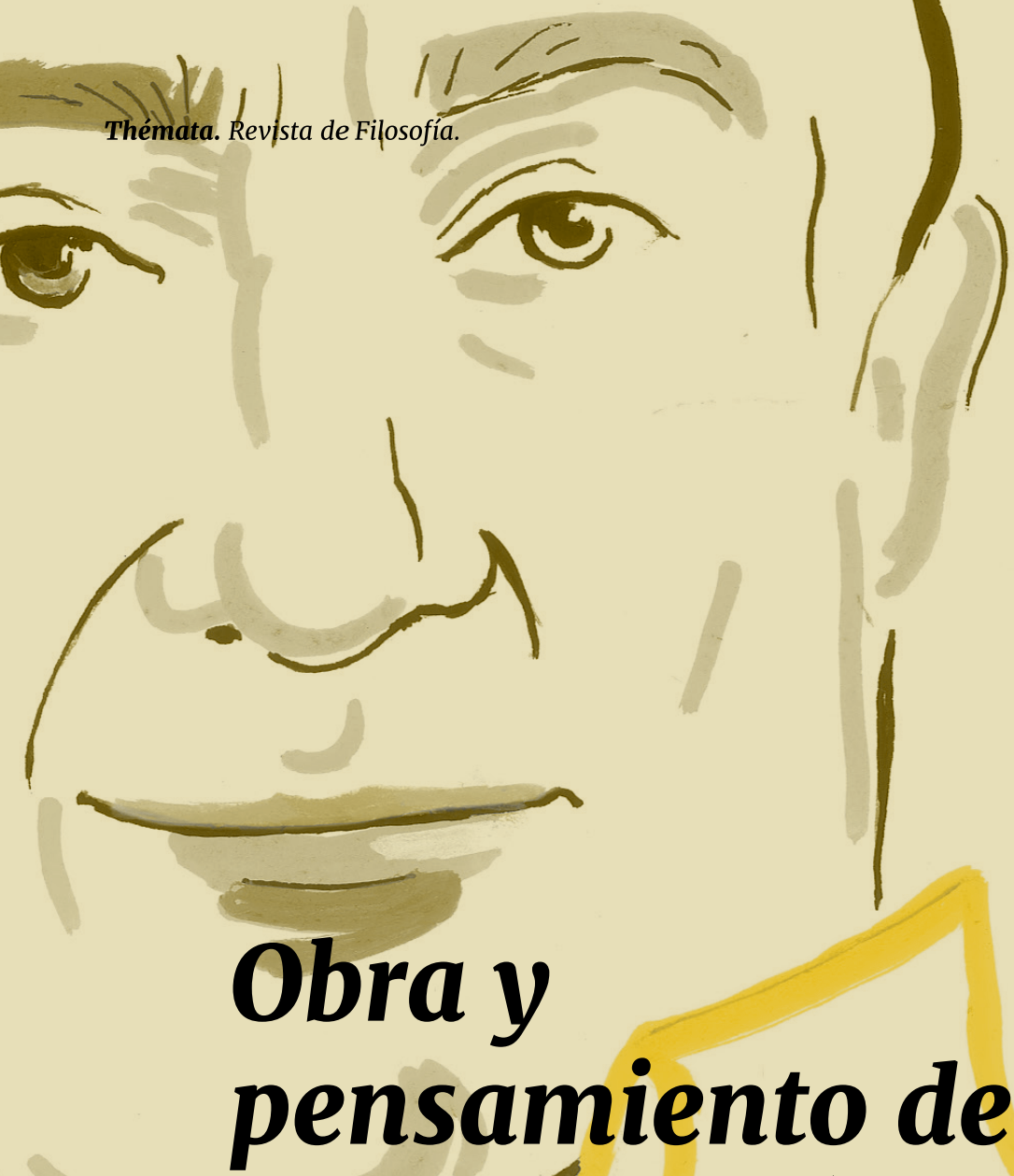

Javier HernándezPacheco Sanz. 


\title{
Una conversación pendiente.
}

A pending conversation.

\author{
Jesús de Garay Suárez-Llanos ${ }^{1}$ \\ Universidad de Sevilla, España \\ Recibido 1 octubre 2021 Aceptado 1 diciembre 2021
}

\section{Resumen}

Se presenta una interpretación de la filosofía de Javier Hernández-Pacheco, al hilo de su confesado aristotelismo. Sus planteamientos quedan resumidos en una filosofía de la reflexión, de la identidad y del deseo. El ser es concebido como sustancia y como actividad. No obstante, todos los planteamientos de Javier Hdez.-Pacheco se inscriben en una filosofía de la libertad, que es concebida como la forma más alta de reflexión y subjetividad. Esporádicamente se mencionan algunos puntos sobre los que el autor y Javier más abiertamente han discrepado: en particular, se discute la primacía de la identidad sobre la diferencia y la libertad.

Palabras clave: Javier Hernández-Pacheco, reflexión, identidad, sustancia, libertad

\section{Abstract}

An interpretation of Javier HernándezPacheco's philosophy is presented, in line with his self-confessed Aristotelianism. His approaches are summarised in a philosophy of reflection, identity and desire. Being is conceived as substance and as activity. However, all of Javier Hdez-Pacheco's approaches are part of a philosophy of freedom, which is conceived as the highest form of reflection and subjectivity. Some points on which the author and Javier more openly disagreed are sporadically mentioned: in particular, the primacy of identity over difference and freedom is discussed.

Keywords: Javier Hernández-Pacheco, reflection, identity, substance, freedom 


\section{Aristotelismo: acuerdos y desacuerdos}

Hace unas semanas terminé un breve artículo sobre la filosofía de Javier Hernández-Pacheco en el que analizo su aristotelismo y su concepto de reflexión. Mis consideraciones se apoyan principalmente en dos de sus libros, la Metafísica (2019) e Hypokeímenon (2003). En ese escrito procuré no añadir mis propios puntos de vista sobre sus planteamientos, sino por el contrario mostrar la coherencia de su sistema filosófico.

Todavía tengo vivo el impacto que me ha supuesto descubrir la profundidad y rigor de sus argumentaciones, ocultas frecuentemente bajo el tono desenfadado y no convencional de sus afirmaciones. A lo largo de los años, había leído algunos de sus libros y siempre le había felicitado por su brillantez literaria y por la originalidad de sus interpretaciones. Pero ahora valoro además la coherencia de sus ideas. Aunque reconocimiento no implica asentimiento. Junto a numerosos puntos de coincidencia, hemos exhibido nuestras discrepancias en más de una ocasión.

Nuestros largos años de amistad han sido también años de largas conversaciones en los que aprendí mucho junto a él. Apenas unas semanas antes de su fallecimiento, habíamos acordado reunirnos en un seminario mensual junto con aquellos profesores y estudiantes que manifestaran interés en trabajar sobre metafísica y sobre Aristóteles. No ha podido ser.

Pero por mi parte deseo ahora continuar esa conversación interrumpida. Es decir, mi intención aquí es examinar algunos aspectos de la filosofía de Javier Hernández-Pacheco, pero sin ocultar mi propia posición, en ocasiones coincidente, en ocasiones discrepante. Pacheco merece que se le tome en serio y eso exige adentrarme también en los terrenos en los que no encuentro suficiente claridad. De esta confrontación espero dos cosas: ante todo, tratar de presentar con mayor viveza sus ideas; y en segundo lugar, de modo un tanto egoísta, aprovecharme del análisis de sus ideas para obligarme a precisar las mías.

Desde luego comparto su aprecio por Aristóteles, así como algunas de sus interpretaciones del aristotelismo. También suscribo su insistencia sobre la unidad de la tradición filosófica. Siempre nos han disgustado esas interpretaciones de la historia de la filosofía en las que se desprecian etapas 
enteras de la historia. Ni aquellas que recelan de la filosofía moderna por su subjetivismo o relativismo, ni las que ignoran la filosofía medieval por su trasfondo teológico. Asimismo siempre hemos valorado la metafísica como el núcleo de toda reflexión filosófica. Quienes abandonan el examen de las preguntas metafísicas, es porque han asumido ingenuamente inconfesadas hipótesis metafísicas. Hay mucho candor en el desprecio de la metafísica.

Sin embargo, habitualmente hemos buscado en direcciones distintas. Pacheco ha intentado integrar la tradición filosófica alemana contemporánea (de Kant en adelante) dentro de la tradición aristotélica, mientras que yo me he centrado en analizar las fortalezas y debilidades de esa misma tradición aristotélica. Obviamente las consideraciones de Pacheco ofrecen un horizonte mucho más vasto y sintético que el mío, ya que ha buscado deliberadamente la confrontación del aristotelismo con la práctica totalidad de la tradición filosófica.

Los dos nos hemos sentido siempre aristotélicos aunque nuestras interpretaciones de Aristóteles fueran frecuentemente dispares. Así, aunque comparto su aprecio por el aristotelismo de Tomás de Aquino, hay puntos en los que me encuentro más cerca del de Escoto, particularmente en su concepción de la libertad y la diferencia. En mi opinión, muchas de las doctrinas en torno a la libertad que Pacheco encuentra y valora en Kant, Fichte o Nietzsche están en continuidad con el aristotelismo escotista, no con el tomista.

Sea como sea, entre las diversas enseñanzas que Pacheco extrae de Aristóteles hay algunas que asumo por entero. Como es sobradamente sabido, Aristóteles afirma que el ser se dice en muchos sentidos: según las categorías de substancia y accidente, como esencial y accidental, como verdadero y falso, y también según el acto y la potencia. Por supuesto, las interpretaciones sobre esta doctrina aristotélica son innumerables e incluso contradictorias. Entre esa diversidad de interpretaciones, Pacheco apuesta por el acto como hilo conductor de los sentidos del ser. Es decir, interpreta el resto de significados del término 'ser' como aspectos diferentes del acto. Y cuando Pacheco dice 'acto', quiere significar actividad, movimiento, acción: entelecheia y energeia. Ser es primariamente acto, actividad. Y desde aquí, le resulta muy cómodo seguir a Tomás de Aquino en su aristotelismo, ya que, como es sabido, el primer sentido del ser para el Aquinate es el acto de ser, 
esto es, el ser como acto ${ }^{2}$.

Acerca de esta interpretación de Aristóteles, he de reconocer que en parte la he defendido también yo en mi libro Los sentidos de la forma en Aristóteles (1987). Sin embargo, ya en ese momento reconocí que los textos para apoyar esa interpretación no son determinantes. Es decir, si nos atenemos a lo que dicen los textos de Aristóteles, esta interpretación centrada en el acto como actividad debe ser completada y matizada en muchos puntos. Una de esas precisiones, a mi modo de ver, se refiere a la esencia y a la diferencia: en Aristóteles 'ser' significa ciertamente acto, pero muy en primer lugar significa esencia, diferencia e identidad. En cualquier caso, estas observaciones son irrelevantes para acotar la filosofía de Pacheco, ya que él era consciente de la originalidad de sus interpretaciones. Buscaba la verdad, no repetir a Aristóteles.

Por otra parte, Pacheco ha reivindicado desafiante el sustancialismo aristotélico. Es conocido el debate en torno a la traducción del término griego ousía. Sin entrar en la controversia, él traduce directamente por substancia. Con ello, desea subrayar dos rasgos de la ousía: la identidad y la subjetividad. Y sin duda en Aristóteles hay una firme defensa de la identidad como elemento esencial de lo real, por lo que si substancia significa identidad, entonces traducir ousía por substancia es completamente legítimo. La realidad ofrece una identidad que además hace posible la ciencia. En el ser hay acto, pero en primer lugar hay identidad: estabilidad, regularidad, continuidad. Todo ello está implicado en el concepto de substancia. Por eso, Pacheco es beligerante ante cualquier propuesta que excluya la identidad.

Por lo mismo, a pesar de su concienzudo estudio de los escritos de Heidegger y Nietzsche, nunca se sintió cómodo con ellos. Y por supuesto, permaneció siempre sordo a los cantos de sirena posmodernos. A menudo me manifestó su disgusto al comprobar mi interés y aprecio por unos y otros.

2 Esta centralidad del ser como acto y como actividad alcanzó un fuerte impulso dentro de la tradición aristotélica gracias al neoplatonismo. En Plotino ser es primariamente actividad, o mejor potencia activa: fuente de actividad. Identidad que se autoconstituye por su actividad propia, al modo de la actividad de los seres vivos, que realizan su identidad mediante la constancia de su actividad. Tomás de Aquino es deudor de ese aristotelismo neoplatónico, especialmente a través de la mediación del liber de causis, el Pseudo Dionisio y Boecio. 
Medio en broma medio en serio me etiquetaba de posmoderno (que en su boca no era en absoluto un elogio). En mi descargo, ya le respondí en más de una ocasión que nunca he puesto en duda la identidad de lo real, y que en este punto me encuentro a gusto en el aristotelismo. Negar la identidad comporta negar la ciencia y el saber y, lo más grave, hace imposible la acción humana.

Donde Pacheco tropieza con los textos de Aristóteles es en su interpretación del ser como sujeto (hypokeímenon), que él además caracteriza como subjetividad. Las Categorías le ofrece un indudable punto de apoyo para su interpretación, porque ahí queda definida la sustancia como sujeto de predicación. Las primeras sesenta páginas de su Metafísica están llenas de atinadas consideraciones al respecto. Ser en primer lugar es sujeto y no predicado. Sin duda. Nada que objetar. Pero contra su interpretación están las observaciones de Aristóteles (Metafísica VII-2) quien, aceptando que la sustancia es sujeto, añade que la substancia no puede identificarse con el sujeto porque entonces la sustancia se identificaría con la materia. En síntesis, que sustancia es sujeto pero no sólo sujeto: ousía es hypokeímenon pero no sólo hypokeímenon. De nuevo debo decir que estas argumentaciones apenas tienen interés para Pacheco, porque (si llegara a convencerle) respondería: allá Aristóteles con lo que dijo o dejó de decir.

En cualquier caso, su interés por la noción de sujeto -además de reforzar la identidad- le permite fortalecer su convicción acerca de la lógica de lo real. Todo lo real es inteligible, lógico. Lógos, physis y arkhé (alma, mundo y Dios, en su planteamiento) forman un trípode en el que se asienta nuestra visión de lo real. Nada carece de lógos. Incluso la teología de Pacheco reivindica continuamente la analogía, es decir, una cierta continuidad entre el lógos humano y el divino. Por todo esto, caracterizar el ser como sujeto -y como sujeto de predicación lógica- implica una clara apuesta en favor de la discursividad lógica humana. Pensar lo real es posible gracias a la predicación lógica: ti kata tinós, decir algo de algo, decir un predicado de un sujeto.

Pero Pacheco no sólo argumenta en favor de la substancia como sujeto. Sino además en favor de la substancia como subjetividad, es decir, como inteligencia reflexiva ("reflexión" en la terminología de Pacheco). En este paso es visible su cercanía a la interpretación hegeliana de Aristóteles. Es sabido cómo Hegel reconoce el Espíritu Absoluto en la noeseos nóesis de Aristóteles (Metafísica XII, 7-9). Caracterizar a Dios como Intelección 
de Intelección es además común al aristotelismo medieval y en particular a Tomás de Aquino. Así, tanto Hegel como el Aquinate parecen remar a su favor. Todo lo real no es sino una manifestación de un Espíritu reflexivo.

El problema es que no hay textos en Aristóteles que avalen la atribución de reflexión a todo lo real. Porque según Pacheco, ser es sustancia, sustancia es sujeto, y sujeto es reflexión. Este paso es el que menos se puede documentar en los textos aristotélicos. Pero ya conozco la respuesta de Javier: si no está en los textos, peor para los textos. Nunca ha ocultado su libertad hermeneútica.

Una última observación respecto al fin. La teleología juega un papel central en todo el planteamiento de Pacheco. Porque es el fin el que hace posible la identidad en el movimiento. El ser es entelékheia: es actividad que tiene ya el fin desde el inicio hasta el término de su actividad. En Aristóteles éste es un rasgo propio de los seres vivos. El ser vivo se mueve por sí mismo. Pero los seres sin vida no. El movimiento de los seres sin vida presenta en los textos aristotélicos otras características. La tradición aristotélica incluso ha fijado la doctrina de que todo lo que se mueve es movido por otro, hasta el punto de que es una de las premisas de las argumentaciones tomistas para la demostración de la existencia de Dios.

En resumen, en la medida en que el universo está lleno de seres sin vida, en esa medida resulta difícil atribuir al cosmos la teleología de los seres vivos. Y eso no sólo en el cosmos aristotélico sino también en el universo concebido por los físicos en la actualidad. El fin del cosmos aristotélico es una unidad de orden: la inteligibilidad del cosmos radica en su orden, esto es, en la subordinación de unos elementos a otros. Y ese orden depende de ese Dios noeseos nóesis. Pero no encuentro argumentos para afirmar que el movimiento de un ser sin vida procede del fin para el que ha sido diseñado. A pesar de sus críticas, creo que la concepción de Ockham de la ciencia física es más creíble (y está más cerca de la posición aristotélica).

En lo que sigue, deseo centrarme exclusivamente en dos puntos. Aunque en realidad es uno solo. Por un lado, quiero mostrar cómo la filosofía de Pacheco pivota sobre la identidad. Interpreta el ser como reflexión. Pero reflexión implica identidad, pues reflexión es volver sobre lo mismo. Lo mismo siempre está ahí para desplegarse, manifestarse o para ser entendido. Y si todo lo real es reflexión, entonces lo real se define por la identidad. Por 
otro lado, querría analizar hasta qué punto su concepto de libertad excluye la novedad. Es decir, en qué medida la acción libre es o no es capaz de innovar diferencias nuevas. Ambas cuestiones están directamente relacionadas, puesto que si la identidad define lo real, entonces resulta difícil la aparición de diferencias nuevas.

Mi interés sobre estas cuestiones toca directamente el núcleo de nuestras discrepancias. Por eso, espero que, al contraponer una posición a la otra, aparezca con mayor claridad la tesis de Pacheco. Ya adelanto que su posición es muy sólida y además goza de una tradición fuerte a su favor. Y desde luego, no pretendo hacer una crítica de sus ideas (que sería de mal gusto, por cierto, ahora que no me tiene cerca para responderme), sino ahondar en la coherencia de su planteamiento. Aparte de otras referencias, me centraré en particular en el capítulo de la Metafísica (pp. 269-335) que lleva por título: De bono. La pasión de existir. En esas páginas creo que se puede encontrar el núcleo de sus ideas acerca de la acción humana y de la libertad.

\section{$2 \cdot$ Identidad y reflexión}

La filosofía de Pacheco es una filosofía de la identidad. Si todo lo real -y Dios en primer lugar- es reflexión, entonces todo vuelve sobre sí mismo, y por lo tanto todo siempre permanece en la identidad de sí mismo. Reflexión es vuelta a lo que ya estaba ahí desde el principio. "Dios existe como una actividad pura de ser que subsiste en su absoluta mismidad como reflexión infinita"3. Difícil encontrar novedad en un Dios que se define como reflexión y como identidad. Pensar la novedad en Dios no ha sido cuestión en Pacheco.

En la medida en que algo no es idéntico consigo mismo, lo es por su carencia de realidad, por su no ser. El subtítulo de su Metafísica es ilustrativo: "O de cómo entre el ser y el no ser, se dan todas las cosas". Es decir, en el principio está Dios, reflexión absoluta (noeseos nóesis), identidad plena, Ipsum esse subsistens. Y más allá de Dios, está el no ser, la inidentidad, la reflexión impedida por la materia y la potencia ${ }^{4}$. Dios está siempre enteramente

3 Met., p. 245.

4 "Esta posibilidad de no ser que la contingencia implica, es más que una posibilidad lógica, y tiene que ver con lo que a lo largo de estas páginas hemos denominado iniden- 
manifiesto a sí mismo. Y en tanto que la verdad es manifestación, Dios es la Verdad siempre idéntica consigo misma. En el universo físico hay tiempo, en Dios eternidad. En nosotros la verdad se va descubriendo paulatinamente en el tiempo, pero siempre está ahí ya -la misma siempre- para ser reconocida.

Las ideas de Pacheco sobre la acción humana hay que comprenderlas en el marco de su afirmación de la identidad de la verdad. Dedica un capítulo entero de la Metafísica (De veritate. La manifestación de lo real, pp. 175-268) a presentar su concepción de verdad. Existe la verdad. Hay una verdad sobre el mundo y sobre lo real. La verdad está ya dada. Y se encuentra en Dios, Reflexión por antonomasia, que tiene presentes y manifiestas todas las cosas, tal como son en sí mismas. En Dios está manifiesta la verdad total de cada ser y su relación con el resto de seres.

Ciertamente en nosotros la verdad sólo está como fin. Hay una verdad de nosotros mismos que Dios conoce, pero que el ser humano sólo puede reconocer como fin de su naturaleza. La identidad está en el fin. Pacheco defiende con toda energía que el ser humano tiene una identidad, una naturaleza, una substancia. Y que hay identidad porque hay fin. Existimos en el tiempo y en la historia, no en la eternidad, por lo que el fin no está nunca plenamente logrado. La verdad de Dios sólo será mi verdad en el juicio final. Por lo demás, estas mismas consideraciones valen para el conjunto del universo físico, más allá del ser humano.

Todo lo real es inteligible y puede ser reconocido en su verdad, porque physis y lógos se corresponden en Dios (arkhé). Pero ese reconocimiento sólo se alcanza en la historia. Mientras, queda el deseo (órexis) 5 . Deseo del fin, deseo de verdad, deseo de identidad. "Toda órexis es así una reflexión, y el Streben que Fichte describe como la esencia del Yo, resulta de que el sujeto es ya originalmente la infinitud a la que aspira"6. El dinamismo natural procede de la fuerza del deseo. Deseamos nuestro fin, el fin que naturalmente

tidad ontológica, identificándola con la idea platónica de materia o la aristotélica de potencia. Las tres cosas (materialidad, potencialidad y contingencia del ente) significan lo mismo. [...] La contingencia, que resulta en el ente de ese límite (no-ser) que lo separa de sí mismo, también se supera conforme el ente realiza su actividad" (Met., pp. 328-9). 5 Hay una errata frecuente que deberá ser corregida en ediciones futuras: hóresis por órexis (ǫ̣̋ $\xi ı$ ): deseo. Por lo demás, Pacheco prefiere usar el término 'voluntad' antes que 'deseo'.

6 Met., p. 272 
tenemos, el fin dado por Dios. Ese deseo de fin es el que pone en movimiento toda nuestra actividad. El deseo del fin define nuestra identidad porque el fin está ya anticipadamente en cada uno de nosotros. No es un deseo de lo ajeno sino deseo que culmina en el fondo de nosotros mismos: es deseo de la propia identidad.

Voluntad es deseo, órexis. Deseo de la identidad que falta y afirmación de la identidad que ya se tiene.

Podemos entender la voluntad como autoafirmación o en general como re-fuerzo de la posición absoluta en que consiste el ente como substancia. Ser es querer ser sí mismo. Querer y ser no se entienden como verbos uno sin el otro. Querer es querer que algo sea, o que no deje de ser. Podemos también decir que ese querer es una reafirmación del ser, en el sentido de que la afirmación de sí que compete al ente en cuanto tal ("A es A") parece quedar reforzada con ese verbo "querer" ("A es y quiere ser $\left.A^{\prime \prime}\right)$. [...] Ser es originalmente un querer ser.7

Dios es Identidad reflexiva total. "Aquí sostenemos que el ser se identifica con el pensar allí donde este último verbo, en la voz activa, expresa el grado máximo de reflexividad subjetiva, en el sentido anteriormente expuesto según el cual ser es vivir y pensar". Pero yo no soy Dios sino reflexividad finita. Por eso, más adelante precisa esta afirmación: "Ser y pensar [...] deberían ser lo mismo. Que no lo sean, la necesidad de distinguirlos, o mejor de cuestionar su "adecuación", es signo de que se trata de una reflexión limitada" 9 . Es decir, ser y pensar se identifican en abstracto. O mejor, en Dios. Pero en nosotros -y en la Naturaleza en general- no hay esa identidad plena, sino sólo una identidad buscada, deseada, querida. "Las cosas quieren ser así más que cosas, y objetos, quieren llegar a ser sujetos" ${ }^{10}$. Las cosas desean alcanzar el fin, es decir, volverse enteramente sobre sí mismas. En eso consiste la finitud: tener la identidad pero no ser la identidad, sino desearla. "Es signo de nuestra finitud que las reflexiones en 
que consisten sujeto y objeto no se cierren en una" ${ }^{11}$.

Lo que en Dios es identidad absoluta, en nosotros es sólo identidad deseada. Pero el deseo de identidad puede realizarse porque Dios está en el fondo de mí mismo: buscar la identidad de mí mismo es buscar a Dios. Este modo de hablar no debe engañarnos porque cualquiera que haya tratado a Pacheco sabe que era alérgico al misticismo ${ }^{12}$. La búsqueda de Dios dentro de sí se orienta a la búsqueda de la propia identidad. Ser uno mismo. Autenticidad. Afirmación de sí, afirmación del propio fin. Así comienza el parágrafo \&135 Seréis como dioses: "El primer mandamiento, el fin de toda actividad, lo que todo ente pretende, es ser; simplemente existir, vivir. Y tratándose de una actividad reflexiva, hay que añadir: por sí mismo" ${ }^{13}$.

El punto de apoyo lo encuentra en Tomás de Aquino. Lo más propio de cada ente es la identidad de su ser (el ipsum esse: su ser mismo), su existir, que le hace ser él mismo, y que ha sido recibido de Dios. "El ipsum esse, ahora in aliquo receptum, nos lo encontramos ahora en las cosas finitas. Y en ellas es también el principio de su reflexividad [...]. El puro ser es, pues, en las cosas, el origen de su reflexividad lógica. [...] El ipsum esse lo encontramos también como actividad reflexiva en todo ente capaz de recoger en sí las relaciones que lo determinan" ${ }^{14}$. En estas consideraciones acerca del Aquinate están algunas claves del pensamiento de Pacheco: 1) El ser es recibido de Dios ${ }^{15}$. De este modo, aleja cualquier sospecha de identidad entre el ser creado y el ser de Dios. 2) Ser es reflexión, que sólo culmina cuando se establece la verdadera relación de todo con todo: es decir, cuando mi reflexión se integra en la reflexión de Dios.

No puedo ir más allá para precisar cómo interpretaba Pacheco la diferencia e identidad de Dios con el mundo y el ser humano. Necesitaría estudiar mejor sus escritos sobre la teología cristiana. Sobre todo porque me encuentro confuso ante afirmaciones como las que terminan el

11 Met., p. 230.

12 Met., p. 290: "Yo, lo siento, nunca he entendido la mística".

13 Met., p. 284.

14 Met., p. 245.

15 "La propuesta que hago en estas páginas consiste en entender que el ser es algo gratis datum, esto es, la radical donación de nuestra mismidad, de aquello que nos hace por sí y no por otro. [...] Como señala Kant: ser sí mismo, es para nosotros un deber. Yyo añado: más bien, una gracia” (Met., p. 330). 
Hypokeímenon: "Para conocer a Dios, y así las cosas, tendríamos que conocernos también a nosotros mismos, del todo, traer al juicio discursivo eso que llamamos Yo, de lo que nos creemos tan seguros porque acompaña como pensamiento todas nuestras representaciones, pero que nunca puede ser representado, porque es tan infinito como esa síntesis total, siempre buscada e inalcanzable como Ideal de la razón, en las que al final el mundo, el alma y Dios serían manifestación de lo mismo"16. Lo que aquí no encuentro explicitado y me gustaría encontrar en otros lugares es cómo interpretaba la diferencia entre yo y el Yo divino. No tanto el yo finito, envuelto en el tiempo y la materia, sino ese yo que ha alcanzado la reflexión total (lo que la teología cristiana denomina "visión beatífica"), pero que sigue siendo un yo finito.

\section{3 - Libertad e identidad}

La posición de Pacheco respecto a la libertad es bastante clara. Bastan unas cuantas citas. "Libertad es tarea, trabajo y esfuerzo, por el que el ente -especialmente el hombre- pretende llegar a ser lo que él mismo es"17. "Esta acción libre, que tiene ella misma la forma de la reflexión"18. "Ser libre, ser sí mismo y obedecer a Dios en el recuerdo de lo que en verdad somos, es todo la misma cosa"19. "La moral es la transformación que hace la libertad de la conducta hasta hacerla expresión dinámica de la propia identidad" ${ }^{20}$. La existencia es un "proyecto de autodeterminación" ${ }^{21}$. Cada uno de nosotros se define como una "pasión de ser sí mismo, que estructura moral y

16 Hyp., p. 445. Cfr. también Hyp. p. 384: Hay "una exigencia de síntesis al final de la cual Dios, Yo y el mundo somos una misma cosa". Asimismo Hyp. p. 373: "Conocerse a sí mismo, y a todas las cosas, es conocer a Dios. Porque Dios no está en la inmensa extensión (como en Newton, para quien el espacio es sensorium Dei), sino más bien en la no menos infinita reflexión que designamos cuando decimos "Yo"'. O también Met., p. 213: "No soy panteísta. [...] Tampoco le tengo miedo filosóficamente al panteísmo. Porque, si bien entiendo que las cosas no son "parte" de Dios, Dios sí es el "Todo" substante de las cosas, en el sentido de que ninguna limita la Divinidad, ni es propiamente un "otro" frente a ella".

17 Met., p. 279.

18 Met., p. 278.

19 Hyp., p. 413.

20 Met., p. 305.

21 ibid. 
orgánicamente la acción humana como un mandato práctico" 22 . "El deber es la autodeterminación de una acción reflexiva. [...] Cada ente debe ser aquello que tiene que llegar a ser" ${ }^{23}$.

Las citas se podrían multiplicar. La libertad es concebida en términos de autoafirmación. Actuar libremente es actuar por sí mismo, es decir, realizar el fin propio. Actuar por uno mismo es actuar conforme al propio fin. De este modo "la libertad es el último fin del hombre" ${ }_{24}$. "Sólo el hombre libre es bueno" 25 . Expresamente señala que la libertad no es sólo condición de posibilidad de los actos buenos para poder elegir, sino que sólo "son buenos aquellos actos en los que la voluntad se determina por sí, y malos aquellos actos en los que la voluntad queda disminuida" ${ }^{26}$. La moral empieza en la libertad y termina en la libertad.

En consecuencia, moral, libertad e identidad se corresponden. Ésta es su "interpretación kantiana de la ética aristotélico-tomista" 27 . "Es "moral" aquello que no tiene otra causa que sí mismo"28. "La acción moral es indistinta de la acción libre: es moral la acción que tiene en sí, y no en otra cosa, su propio principio. Por eso, el que obra moralmente no puede hacerlo de otra forma: su acción y su ser son lo mismo" 29 . Pacheco no escribía estas ideas como un juego especulativo sino que vivía comprometido con ellas. Cuántas veces le hemos oído decir: "no puede ser de otro modo". No por la fatalidad del destino, sino por ser expresión de la propia naturaleza.

Resulta muy ilustrativo su concepto del mal. Por una parte, el mal tiene que ver con "una inidentidad o disminución del ente" ${ }^{30}$, una insuficiencia o limitación ontológica. Pero "ese déficit ontológico se convierte realmente en mal cuando es de alguna manera "consentido", de modo que el ente claudica en la exigencia de superar ese déficit que su propia reflexividad ontológica le

22 ibid.

23 Met., p. 292.

24 Hyp., p. 410.

25 ibid.

26 ibid.

27 ibid.

28 Hyp., p. 347.

29 Hyp., p. 348.

30 Met., p. 319. 
plantea" ${ }^{31}$. El pecado consiste en querer "aquello que nos separa de nosotros mismos y de nuestro último fin" ${ }^{32}$. El acento está en el querer, en el querer permanecer en la inidentidad.

De ahí que conciba el mal principalmente como pereza, como renuncia al trabajo en pro de la identidad. "El pecado original sería propiamente la pereza o conformidad del sujeto activo con su circunstancia empírica: acomodarse. El sujeto renuncia a ser lo que verdaderamente es"33. Y de ahí su rechazo al estoicismo o a Spinoza, en tanto implican conformismo con la necesidad de lo real. Si el mal está en querer permanecer en la inidentidad, "el ser bueno bien puede dilucidarse como autenticidad, y el deber como la obligación de ser sí mismo"34.

Este enfoque es asimismo visible en su identificación de la conciencia moral con la autoconciencia. "Hay una cierta tendencia a olvidar que conciencia moral y conciencia cognoscitiva son lo mismo" 35 . "La autoconciencia es un mandato, un imperativo. "Conócete a ti mismo", "llega a ser lo que eres", quieren decir lo mismo; y son las dos formas, teórica y práctica de la (auto-) conciencia" ${ }^{36}$. De este modo, la libertad no sólo es un deseo de identidad sino además es un deseo de reconocimiento, de reflexión. Uno es tanto más libre cuanto más se conoce a sí mismo. En rigor, la acción moral es sólo acción inmanente y sólo actividad intelectual que vuelve sobre sí misma. "La conciencia moral expresa la identidad refleja de esa actividad, ahora como mandato de ser sí misma, de no perderse en la pluralidad de sus determinaciones circunstanciales, sino de autodeterminarse en ellas. Se trata ahora de mantener un proyecto vital de autenticidad en medio del mundo" 37.

De este modo la trascendencia en la conciencia es la trascendencia del fin respecto de la condición actual. “¡No hay derecho!” es la reivindicación de justicia frente a la injusticia actual, de infinitud frente a la finitud, de verdad frente a la falsedad. En nosotros existen ya la justicia, la verdad y la infinitud, 
aunque como fin. Y la tarea es alcanzarla. Ya tenemos la libertad aunque finita: nuestra misión es transformarla en esa libertad infinita a la que estamos llamados. Así se cierra el círculo de la identidad: desde la libertad a la libertad. De la verdad a la verdad. De la justicia a la justicia. Y el motor es el deseo (la órexis) o voluntad: el deseo del fin. Por eso, autoconciencia y conciencia moral quedan identificadas: conocerse es conocer el propio fin. Y al conocerlo, se vive conforme a ese fin. Hay bastante intelectualismo moral a lo Sócrates en Pacheco. Si la actividad más alta es la reflexión, entonces la reflexión plena se traduce en la acción moral por antonomasia. La moral se cifra en la autenticidad.

En esta acción moral no quedan excluidos los demás hombres ni el mundo, pues la autoconciencia sólo es posible si nos reconocemos en nuestra relación con los demás y con el mundo. "La libertad es ese impulso creativo infinito que se extiende a la totalidad de lo que es, como un proyecto de universal transformación en el que se liberan las fuerzas de la naturaleza" ${ }^{38}$. "Esa tarea de ser sí mismo equivale a una infinita expansión del foco subjetivo y se hace así un proyecto universal de transformación" ${ }^{39}$. La voluntad de afirmarse a uno mismo sólo puede realizarse si es simultáneamente afirmación de todo lo real, en tanto que nuestro ser está en relación con todo lo real.

Quiero terminar estas páginas igual que las inicié. Con acuerdos pero también con desacuerdos. En el punto en el que se interrumpió nuestra conversación. En la relación entre voluntad y deseo. Porque, concedo, sin duda existe el deseo de verdad, de libertad y de justicia universal. Y ciertamente esa es tarea del hombre. Y desde luego asumir ese deber moral puede definirse como autenticidad. Hasta aquí no me cuesta seguirte. Efectivamente libertad implica identidad: actuar por sí mismo.

Pero además de todo esto, libertad implica diferencia, novedad. En este punto nunca nos hemos entendido. Pacheco dedica muchas páginas a criticar "cierta tradición filosófica que confunde la voluntad con sus manifestaciones dinámicas, como son el deseo, el esfuerzo, el afán; y en general con aquello que en nosotros provoca inquietud, insatisfacción o inconformidad. En

38 Met., p. 286.

39 Met., p. 279. Cfr. igualmente Hyp., pp. 353-363: "la conciencia moral es conciencia de la propia subjetividad como un "nosotros"” (p. 354). 
este sentido, se ve en el querer una voluntad de cambio y afán de lo otro. [...] Se valora la voluntad primariamente como innovación, como constante reformulación de valores, que resultan de esa voluntad creativa" 40. "Ser es entonces para ese ente la inquietud que busca superar el límite que separa a cada cosa de sí misma", mientras que el deseo propiamente es la "reafirmación o re-fuerzo de lo que originalmente es, y así como resistencia a la descomposición" ${ }^{41}$.

Frente a la antropología de la innovación en la que prima la arbitrariedad infantil, los antiguos valoraban el concepto de virilidad. [...] Se habla en este contexto de "tener los pies en el suelo", de "raíces", de ser "hombre de principios". [...] Podríamos hablar también de una "voluntad asentada". En definitiva, frente a la infundamentalidad de la continua novedad, la voluntad "viril" (virtud por cierto muy femenina en los paradigmas clásicos, como Andrómaca y Penélope) representa en su reflexividad una apropiación del fundamento, de los principios; y esto lo podemos llamar "solidez", pero también desde un punto de vista ontológico, substancialidad de la conducta. [...] Es el sí mismo mantenido como fin (la identidad que subyace al movimiento) lo que distingue la órexis proyectiva, el querer ser, del capricho arbitrario. ${ }^{42}$

Creo que esta larga cita resume perfectamente la visión de la vida de Pacheco. (Y aunque eso ahora importa menos, el fondo de sus críticas hacia mis ideas). En su filosofía y en su vida, la acción queda asentada sobre la identidad del fundamento. Sobre la substancialidad y sobre el fin.

Sinceramente sospecho que, si continuáramos la conversación, querido Javier, podría convencerte de que la voluntad libre es creadora sin ser caprichosa ni arbitraria. Y que la voluntad no es sólo deseo de autoafirmación, sino también creadora de diferencias nuevas. La libertad no es deseo, aunque haya un deseo de libertad. Mi esperanza de que podríamos ponernos de acuerdo está en una afirmación que haces en el Hypokeímenon, 
de connotaciones típicamente kantianas: la experiencia moral, dices, implica "un origen absoluto de actividad, una acción originalmente libre que no debe (...) sucumbir a la necesidad natural" 43 . Aquí afirmas que la libertad no está sometida a la necesidad natural. Entonces, tampoco lo está a la necesidad natural de sí mismo. Tampoco a la necesidad del fin de sí mismo. Por eso quizá aceptaras que la libertad también crea fines, más allá del fin natural. Y puede ser creadora sin ser caprichosa, en tanto no contradiga ese fin natural. Porque la no contradicción funda la racionalidad y nos salva del capricho. La acción libre es racional y creadora porque se despliega desde la no contradicción.

Termino ya. Hay un punto en el que creo que pronto nos pondríamos de acuerdo. El Dios cristiano es un Dios vivo, un Dios que actúa y que lo hace libremente. Un Dios así es un Dios que no permanece idéntico en su reflexión eterna, sino que introduce diferencias nuevas en la vida. Un Dios que sea sólo reflexión e identidad difícilmente puede concebirse como un Dios vivo, al menos tal como el cristianismo entiende a Dios. Por eso, la identidad y la reflexión, por importantes que sean (que lo son), no pueden ser la última palabra. Además hay diferencias: diferencias entre Dios y el mundo, entre Dios y yo, diferencias entre diferentes identidades. Y además hay diferencias creadas por las libertades, que convierten la realidad en una realidad siempre nueva. En fin, aquí se interrumpe nuestra conversación. Quién sabe si en algún momento podremos retomarla. 


\section{Thémata. \\ Revista de Filosofía}

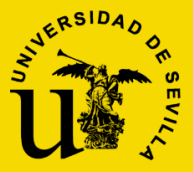

